\title{
Reduction of carbon byproducts for high-purity carbon nanocoil growth by suppressing catalyst collision
}

Takehiro Gohara, Kuniharu Takei, Takayuki Arie, and Seiji Akita*

Department of Physics and Electronics, Osaka Prefecture University

1-1 Gakuen-cho, Naka-ku, Sakai, Osaka 599-8531, Japan

Carbon nanocoils (CNC) are promising nanocarbon materials for application as functional composites such as electromagnetic wave absorbers. However, the suppression of carbon byproducts during CNC growth is necessary to obtain high purity CNCs with high yield. Here, we demonstrate a simple but efficient method for the reduction of carbon byproducts using a patterned thin film catalyst. Results show that the collision of tip catalysts of growing CNCs to the substrate or other CNCs inhibits the smooth growth of CNCs because of the deactivation of the catalyst. The collision also stimulates the formation of a carbon byproduct layer underneath the $\mathrm{CNC}$ layer. The well-controlled thin film catalyst with checkerboard pattern brings us four-times-higher efficiency of the catalyst and suppresses the carbon byproduct layer to $\sim 1 / 3$.

\footnotetext{
* Tel: +81-72-254-9261, E-mail: akita@pe.osakafu-u.ac.jp (Seiji Akita)
} 


\section{Introduction}

Because of their helical structure, carbon nanocoils (CNCs) $[1,2]$ are promising as functional nanocarbons for applications such as nano-mechanical components [3, 4], field emission sources [5-8], and electromagnetic wave absorbers [9-12]. For these and similar applications, mass-production synthesis is indispensable. Many attempts have been made to improve the synthetic yield of CNCs. Thermal chemical vapor deposition (CVD) is commonly used to synthesize CNCs in which catalyst metals play an important role. Therefore, many studies related to catalyst for growth of $\mathrm{CNCs}$ have been conducted to develop highly efficient catalysts for CNC growth based on transition-metal based catalysts, including the use of $\mathrm{Co}, \mathrm{Fe}$, and $\mathrm{Ni}[1,2]$, and multicomponent-catalysts such as $\mathrm{Fe} / \mathrm{Sn}$, $\mathrm{Fe} / \mathrm{In} / \mathrm{Sn}$ alloys [8, 13-16], and K/Ag [17]. Aside from these efforts for the development of catalysts, the interaction between the substrate and catalyst reportedly affects CNC growth [18-20]. The additional Sn buffer layer strongly supports the formation of highly efficient catalytic particles at the very early stage of CNC growth, which results in the high yield synthesis of CNCs. Consequently, the catalyst formation at an early stage is a key parameter to realize the mass synthesis of CNCs.

In addition to the catalyst formation at an early stage, the positioning of the catalyst including the density of catalyst on the substrate must be important. In the case of thin film type catalyst, however, little attention has been devoted to this issue, where the whole substrate was covered with the catalyst layer. The lifetime of the catalyst particle is also important for high yield synthesis. Recently, we investigated the growth of individual CNCs using in-situ optical microscopy to reveal the growth mechanism of CNCs [21]. Results showed that the smooth growth of some CNCs was inhibited by the collision of catalyst particles at the tips of the CNCs. Furthermore, we observed very long CNCs longer than 100 $\mu \mathrm{m}$ at the substrate edge, whereas the average length of CNCs was approximately $10 \mu \mathrm{m}$, 
which implies that a lower collision rate at the edge is preferred for $\mathrm{CNC}$ growth in comparison to the central part of the substrate, where the density of grown $\mathrm{CNCs}$ is higher than that at the substrate edge. Consequently, the position or arrangement of the catalyst is an important parameter to realize an efficient catalyst with a long lifetime.

This study investigated the effects of collision on CNC growth by changing the distance between the catalysts to control the collision probability. We also demonstrated a simple but efficient method for the reduction of the carbon byproduct by controlling the arrangement of catalyst pattern.

\section{Experiments}

Fig. 1 presents a schematic of the catalyst arrangement on a substrate for investigation of collision effects on CNC growth. In this arrangement, we expect that the collision probability of CNC tips between the catalyst stripes can be varied by changing the gap of the catalyst stripe. A narrower gap is expected to be attributable to the higher collision rate because of the presence of the $\mathrm{CNC}$ grown from the counter stripe in addition to the collision to surrounding CNCs on the same stripe. The growth of CNCs on top of the catalyst is also affected by the collision of surrounding CNCs. These effects will be discussed. 


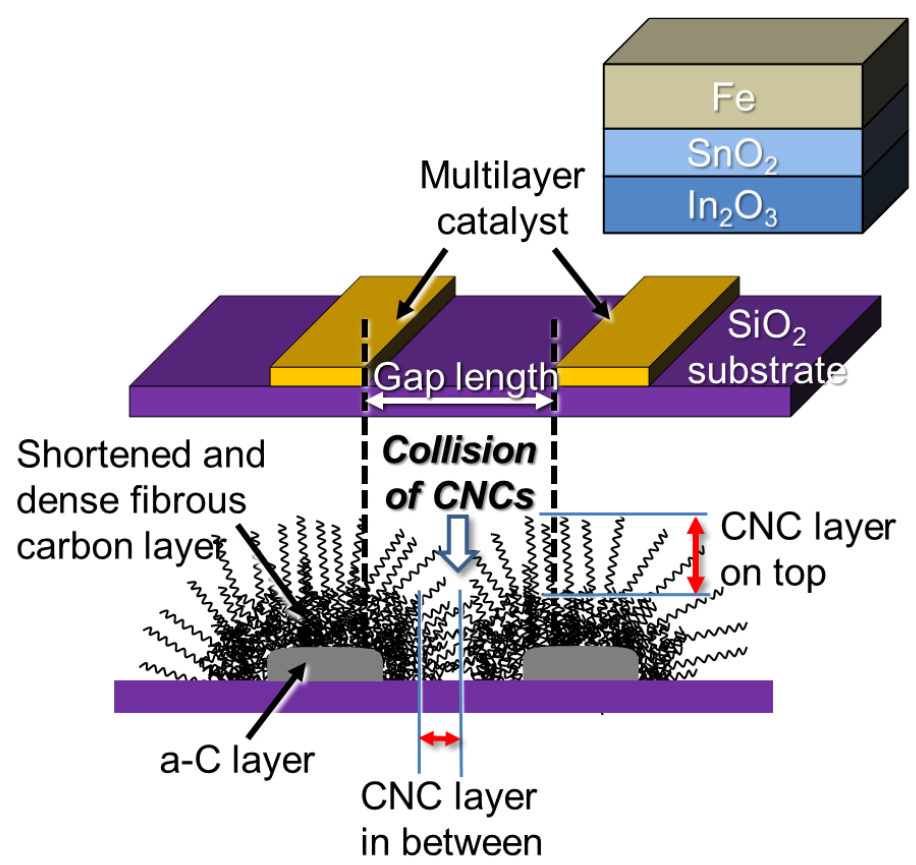

Figure 1(a) Schematic illustration of the arrangement of thin film catalyst to investigate the collision of the growing $\mathrm{CNCs}$, where a tri-layer catalyst was deposited on the $\mathrm{SiO}_{2} / \mathrm{Si}$ substrate. (b) Schematic illustration of cross-sectional view for grown $\mathrm{CNCs}$ around the catalyst.

In this experiment, thin film catalysts were used because they can form a stripe pattern using photolithography, and vary its composition ratio merely by changing each thickness. The multilayered thin film catalysts of $\mathrm{Fe}, \mathrm{SnO}_{2}(\sim 15 \mathrm{~nm})$, and $\mathrm{In}_{2} \mathrm{O}_{3}(\sim 20 \mathrm{~nm})$ were prepared by sputtering deposition on a $10 \times 10 \mathrm{~mm} \mathrm{SiO} 2$ substrate. The $\mathrm{Fe}-\mathrm{Sn}-\mathrm{In}$ catalyst was reported as a high-yield catalyst for $\mathrm{CNC}$ growth [13], where the $\mathrm{Fe}: \mathrm{Sn}=5: 1$ catalyst provides the best results for stable growth of CNCs with small distribution of coil diameters, about 0.5-1 $\mu \mathrm{m}$ (see "supporting information" for optimization of growth condition). After the deposition, the catalyst on the substrate was oxidized at $600{ }^{\circ} \mathrm{C}$ in ambient conditions for 10 $\min$.

The CNCs were grown using atmospheric CVD, using $\mathrm{C}_{2} \mathrm{H}_{2}$ as a carbon feedstock with a flow rate of 5 standard cubic centimeters per minute $(\mathrm{sccm})$, mixed with $50 \mathrm{sccm}$ Ar. CNCs 
were synthesized at different temperatures of $800{ }^{\circ} \mathrm{C}$. To perform in-situ observation of $\mathrm{CNC}$ growth, we use a small reactor (cross-sectional area: $5 \times 20 \mathrm{~mm}$ ) and a furnace with an optical microscope, as described in an earlier report of the literature [21].

\section{Results and discussion}

\subsection{Effect of collision of catalyst particle on CNC tip}

Fig.2(a) presents the gap-distance dependence of the CNC layer thickness between two adjacent stripes measured from the cross-sectional SEM images for each gap, where the reaction time was also varied: 5, 10, 20, and $30 \mathrm{~min}$. The CNC layer thickness between the adjacent stripes increases concomitantly with the increase of the gap. A straight line in Fig.2(a) shows the half length of the gaps. It is readily apparent that the CNC layer thickness between the adjacent stripes for all deposition times is thinner than the half length of the gap, even after the growth of $30 \mathrm{~min}$. It should be noted that the impurity layer at the root of $\mathrm{CNCs}$ is still grown owing to the excess supply of carbon source to the remaining catalyst on the substrate. As a result of the growth of amorphous carbon layer, the roots of CNCs are covered with the amorphous carbon layer. Thus, the total CNC length at $30 \mathrm{~min}$ is shorter than that at 20 min as observed in Fig.2(a). The CNC layer thickness on top of the catalyst layer, which corresponds to the free from the collision from the adjacent stripe, shows no clear gap dependence. It saturates to approx. $55 \mu \mathrm{m}$ after the growth of $30 \mathrm{~min}$, even for the gap of 20 $\mu \mathrm{m}$, as portrayed in Fig.2(b). In the case of no collision of CNCs in the gap, the grown CNC length in between portrayed in Fig.2(a) is almost identical to the thickness corresponding to the $\mathrm{CNC}$ length on top of the catalyst layer. Fig.2(c) presents a series of the temporal variation of in-situ optical microscopy images of $\mathrm{CNC}$ growth on the stripe patterned catalyst. The entangled fibrous carbon layer as presented in Fig. 1 gradually occupies the gap separating the adjacent stripes with the growth and results in no space for CNC growth at the gap in the case 
(a)

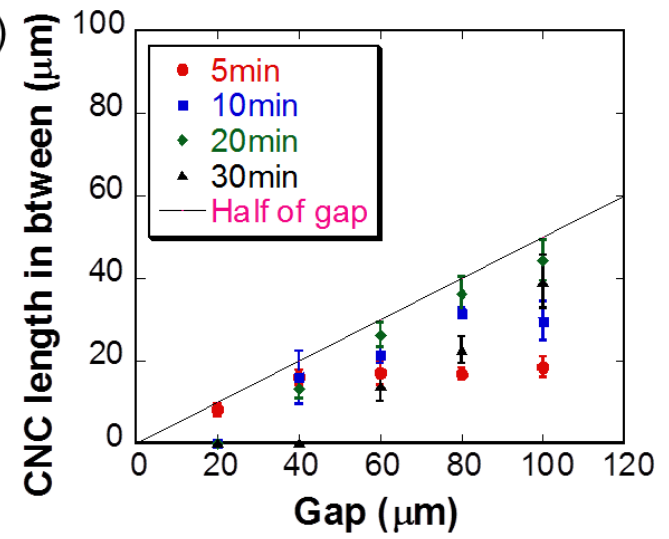

(c) (b)

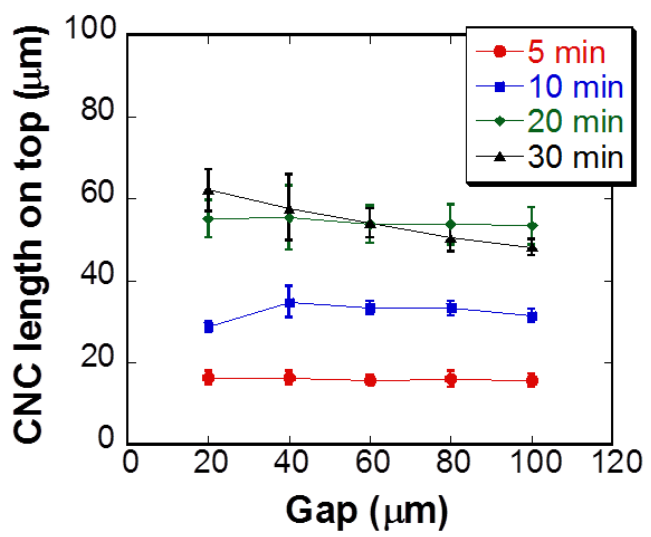

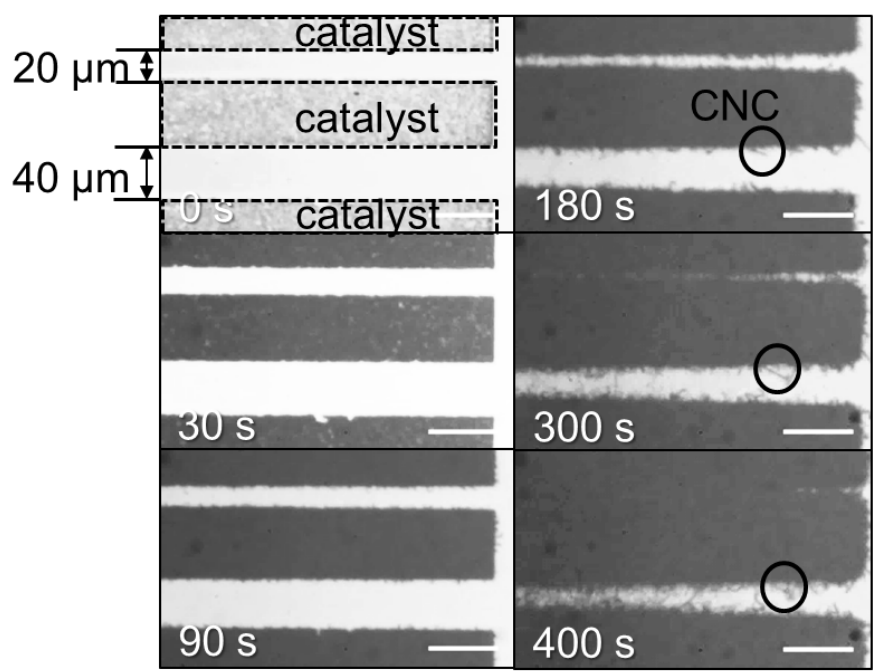

Figure 2 Effect of gap distance dependence on CNC growth: (a) Gap distance dependence of CNC length grown between adjacent strips with several growth time. A straight line represents half of the gap distance. (b) Gap distance dependence of CNC length on top of the catalyst center with several growth time. (c) Temporal variation of the in-situ optical microscope image of growing CNCs around the different stripe gaps with $20 \mu \mathrm{m}$ and $40 \mu \mathrm{m}$.

of gap less than $20 \mu \mathrm{m}$ and the reaction time longer than $5 \mathrm{~min}$ as portrayed in Fig.2(c). These results strongly support the model by which the collision of the catalyst particle on the tip of CNCs inhibits the CNC growth.

Fig.3(a) presents a cross-sectional SEM image of the grown CNCs using stripe catalyst with a growth time of $20 \mathrm{~min}$. A tri-layered structure, shown schematically in Fig. 1, is readily apparent: outermost, middle, and innermost layers are composed respectively by CNCs, 
shortened and dense fibrous carbon layer (Fig.3(b)), and dense carbon layer (Fig.3(c)). Based on the model that the collision of each $\mathrm{CNC}$ inhibits the growth of $\mathrm{CNC}$, the middle carbon layer depicted in Fig.2(b) was formed by the collision of CNCs. Only the survived CNCs form the outermost CNC layers. Results show that the cross section of the shortened and dense fibrous carbon layer is a hog-back shape, i.e., the middle carbon layer around the center is thicker than that edge region, which suggests that the collision probability of CNCs around the center is greater than that around the edge region. As shown in Fig. 3(d), the ratio of CNC layer area in the cross sectional SEM image increases with increasing the gap length to $60 \%$ and saturates around $74 \%$. This result suggests that the elimination of collision of the catalyst particle on the tip of CNCs is efficient to improve the purity of $\mathrm{CNC}$.
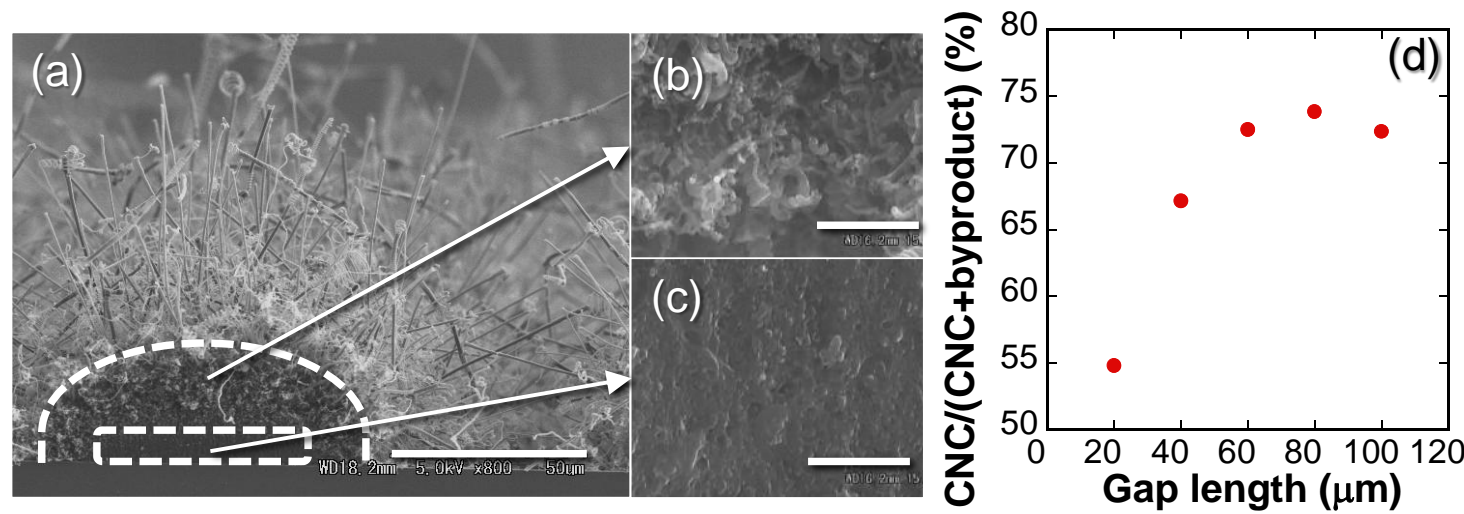

Figure 3 Cross-sectional SEM image of stripe catalyst: (a) SEM image of the cross section around the stripe catalyst (scale bar is $50 \mu \mathrm{m}$ ). (b) Expanded view at the carbon byproduct layer consisting from dense and shortened CNCs (scale bar is 2 $\mu \mathrm{m})$. (c) Expanded view of the carbon byproduct layer consisting of dense amorphous carbon. (d) Gap length dependence of the ratio of areas between CNC and byproduct measured from the cross sectional SEM image.

\subsection{Growth model based on catalyst collision}

Based on the collision model, because the entangled fibrous carbon layer is formed mainly by the collision of the growing CNCs or carbon fibers as described previously, the analysis of 
the formation mechanism of the entangled fibrous layer is important to realize the high-yield synthesis of CNCs. Fig.4 presents the growth model of CNCs. We have reported that the catalyst color was changed suddenly from a metallic color to black with volume expansion when $\mathrm{C}_{2} \mathrm{H}_{2}$ gas had reached the catalysts from the in-situ observation using optical microscopy [21] as presented schematically in Fig.4(a). At this time, $\mathrm{C}_{2} \mathrm{H}_{2}$, the source gas, was decomposed thermally when it reached the reaction area and the dehydrogenation reaction was happened on the catalyst surface. Then the carbon atoms were diffused into catalyst metals, forming the bottom a-C layer presented in Fig.4(b). As presented in Fig.4(b) (see also Fig.3(c)), the bottom a-C layer is a rectangular cross section and much thicker than the initial catalyst thin film. Then, the catalyst particles of $\mathrm{Fe}_{3} \mathrm{SnC}$ or $\mathrm{Fe}_{3} \mathrm{C}$ for growth of CNCs or carbon nanofibers (CNFs) were formed on the surface of the bottom a-C layer. The catalyst particle density on the bottom a-C layer is expected to be high. Although the growth of $\mathrm{CNC}$ is initiated at the catalyst particles as presented in Fig.4(c), the continuous growth of the most of CNCs is stagnated by collisions because of excess density of the catalyst particle. Stagnated CNCs were piled up, forming a shortened and dense fibrous carbon layer on the bottom a-C layer, as depicted in Fig.4(d) (see also Fig.3(b)). This collision process is repeated. The shortened and dense fibrous carbon layer continues to expand as described in Fig.2(c). 
(a) $0 \mathrm{~s}$

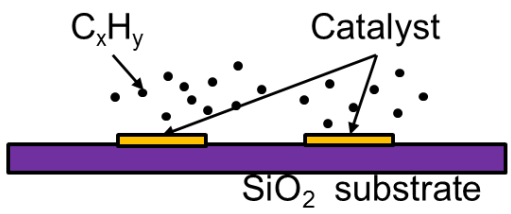

(b) $\sim 10 \mathrm{~s}$

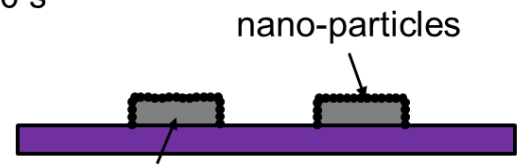

a-C layer initiation of CNC growth (d) $\sim 180 \mathrm{~s}$

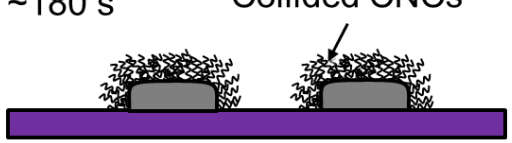

Shortened and dense

(e) $\sim 300 \mathrm{~s}$ fibrous C layer

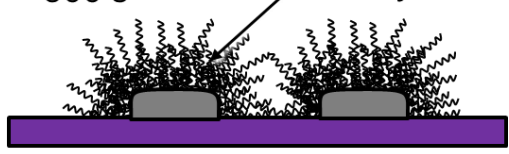

(f) $\sim 400 \mathrm{~s}$

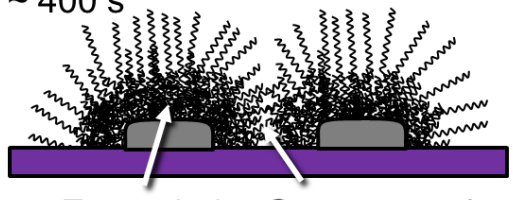

Entangled Gap occupation fibrous C layer

Figure 4 Growth model of CNCs: (a) immediately after the introduction of source gas, (b) nanoparticles and a-C layer formation after $10 \mathrm{~s}$, (c) very early stage of $\mathrm{CNC}$ formation on the catalyst surface, (d) beginning of collision of adjacent CNCs, (e) beginning of formation of shortened and dense fibrous $\mathrm{C}$ layer and of collision of CNCs at the gap, and (f) the gap is occupied by collided CNCs.

The survived CNCs in the collisions continue to grow as presented in Fig.4(e). In this case, the collision probability is much lower than that for the initial growth stage because of the expansion of distance between the growth sites. These CNCs can be observed as filament-like structures grown from the edge of expanded stripe pattern as observed by the in-situ optical microscopy portrayed in Fig.2(c). Long CNCs (longer than 2-3 $\mu \mathrm{m}$ ) begin to vibrate because of increased flexibility, heat, and turbulence of source gases. This vibration causes an increase of the collision probability to the surrounding CNCs. It inhibits the growth of long CNCs, as presented in Fig.2(c). This situation closely resembles the case in which the gap separating the adjacent stripes as discussed in Fig.2. Finally, very few CNCs without collisions are able to grow until the catalyst life time. In this way, the $\mathrm{CNC}$ growth is affected strongly by the collision of others including the substrate at each growth stage. 


\subsection{Suppression of collision of tip catalyst toward mass synthesis}

Figs. 5(a)-5(c) show cross-sectional SEM images for catalyst pattern dependence of grown CNCs, where catalyst patterns are depicted schematically in the insets, (a) fully covered with the catalyst layer, (b) stripe pattern with the width of $20 \mu \mathrm{m}$, and (c) checkerboard pattern with $2 \mu \mathrm{m}$ square with a $2 \mu \mathrm{m}$ gap. The dense carbon layer with $15 \mu \mathrm{m}$ is observed clearly in Figs. 5(a) and 5(b). The thicknesses of "the shortened and dense fibrous carbon layer" are also similar for both cases. At the gap region for adjacent catalysts, the grown CNCs from the edge region are visible. In both cases, the growth directions of CNCs to the substrate are distributed randomly and the grown CNCs are entangled. These characteristics imply that the collision between growing CNCs prevent the smooth growth of CNCs. Consequently, the central region of the $20-\mu \mathrm{m}$-width stripe catalyst is insufficient for suppression of the tip catalyst collisions.

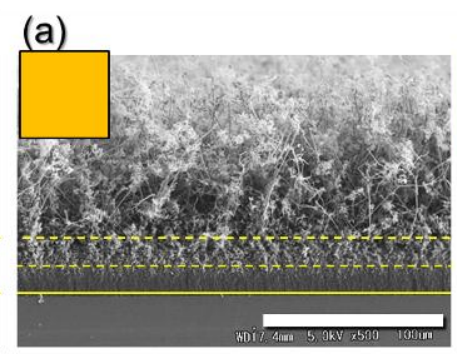

(d)

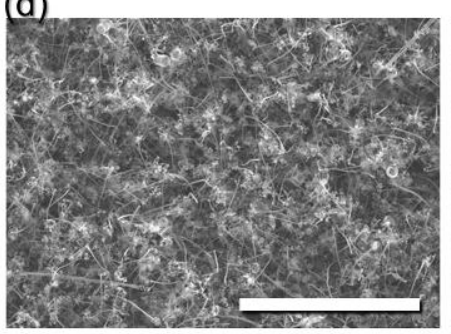

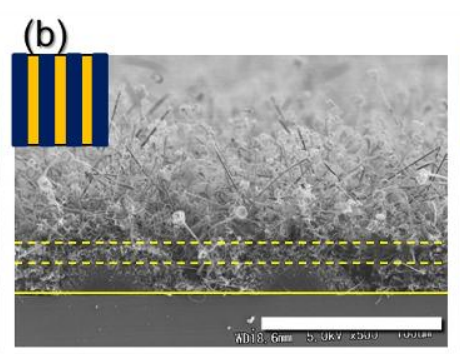

(e)

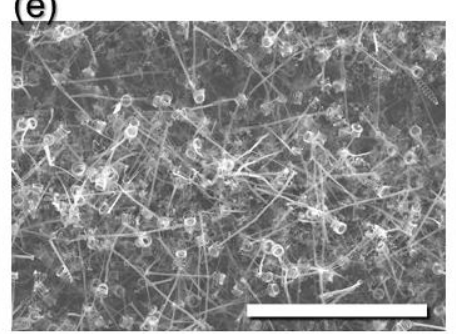

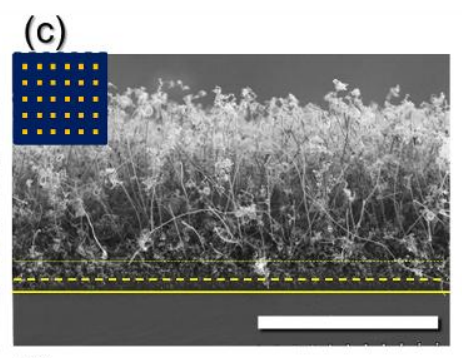

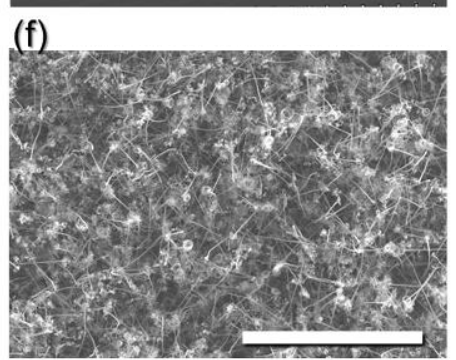

Figure 5 Suppression of dense a-C and shortened and dense fibrous layer: cross-sectional and top view SEM images of CNCs grown from (a) and (d) a substrate fully covered with catalyst layer, (b) and (e) stripe catalyst with a width of $20 \mu \mathrm{m}$ and (c) and (f) checkerboard pattern with $2 \mu \mathrm{m}$ square. Yellow parts are insets of (a)-(c), showing the catalyst pattern on the substrate. All scale bars are $100 \mu \mathrm{m}$. 
In the case of Fig.5(c), the ratio of the circumference length of the square catalyst to the surface area of the square catalyst is much higher than that both for the fully covered and stripe catalyst. This height induces the reduction of the tip catalyst collision at the early growth stage around the edge of the catalyst, which results in the suppression not only of the "shortened and dense fibrous carbon layer" but also of the "dense carbon layer" less than 5 $\mu \mathrm{m}$. Additionally, it is difficult to find a clear boundary separating "the shortened and dense fibrous carbon layer" to the CNC layer. The growth of the CNCs to the substrate is perpendicular to the substrate in comparison with the former cases and the grown CNCs are less entangled and less wavy shape. Furthermore, although the coverage ratio of the catalyst layer to the substrate is only $25 \%$ for the checkerboard pattern, the total thickness of the CNC layer is almost equal to that of the fully covered with catalyst layer depicted in Fig.5(a), which indicates that the catalyst efficiency of the checkerboard pattern for growth of CNCs is four times higher than that for conventional (fully covered with catalyst layer) thin film catalyst. Figs. 5(d)-5(f) respectively present SEM images of top views of Figs. 5(a)-5(c). The CNCs observed in Fig.5(d) shows a wavy shape. In contrast, many CNCs depicted in Fig.5(e) and 5(f) corresponding to the smaller collision probability are observed as straight rods.

We have performed additional experiments to investigate the effect of the gap length for checkerboard pattern. In the case of the wide gap of $24 \mu \mathrm{m}$, the thickness of byproduct layer could be greatly reduced as shown in Fig. 6 and the purity defined by the ratio of areas between the $\mathrm{CNC}$ and byproduct reaches to $88.7 \%$. In this case, however, the wider gap must result in the reduction of CNCs layer thickness as shown in Fig. 6 because of the reduction of the total catalyst area on the substrate, even though the larger gap is effective to reduce the byproduct. For instance, in the case of $3 \mu \mathrm{m}$ square with $24 \mu \mathrm{m}$ gap shown in Fig. 6(c), the catalyst area on the substrate is only $1.2 \%$. Surprisingly, the whole of substrate was covered with $\sim 50 \mu$ m-thick CNC layer even under the condition that the catalyst covers only $1.2 \%$ as 
shown in Fig. 7. The catalyst efficiency for this case is $\sim 50$ times higher than that for fully covered catalyst estimated from the $\mathrm{CNC}$ layer thickness and coverage of catalyst on the substrate.
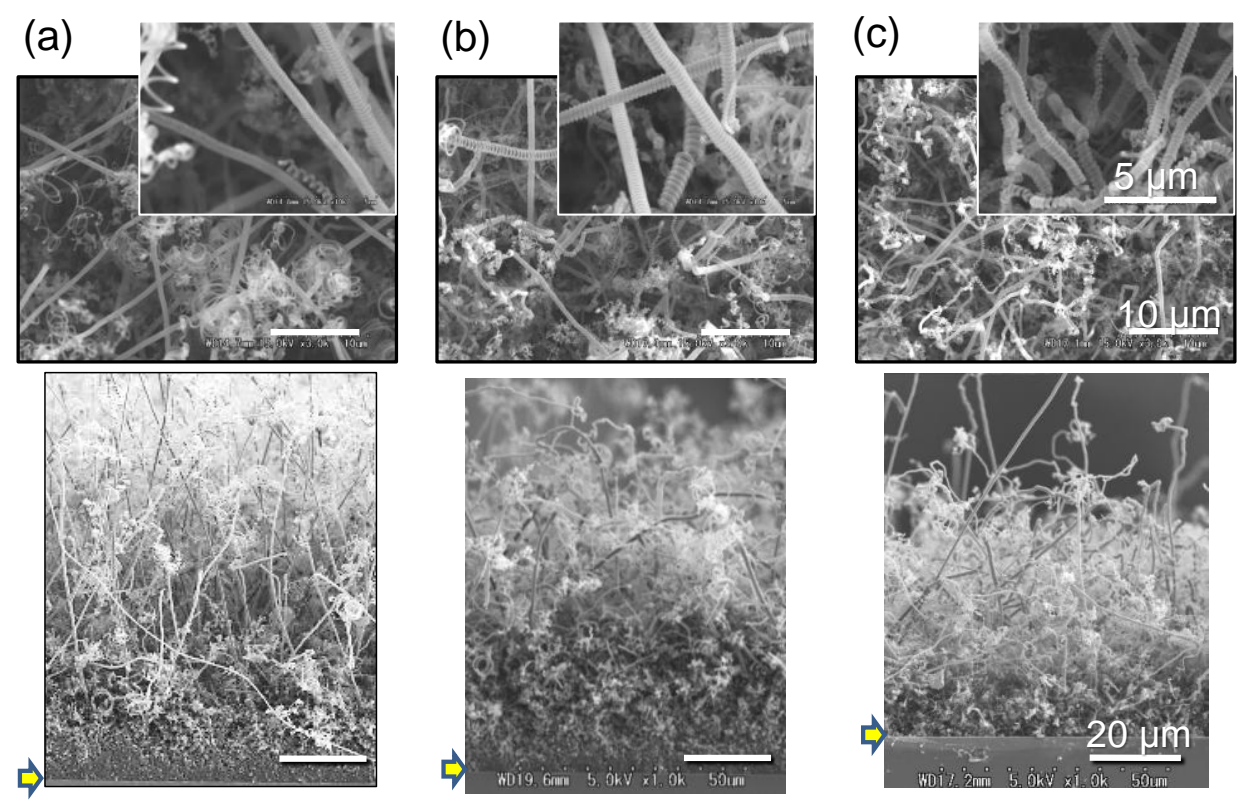

Figure 6 SEM images of grown CNCs with different gaps with (a) $2 \mu \mathrm{m}$, (b) $6 \mu \mathrm{m}$, and (c) $24 \mu \mathrm{m}$ for checker board pattern catalyst. Arrows of bottom images indicate the substrate edges. 

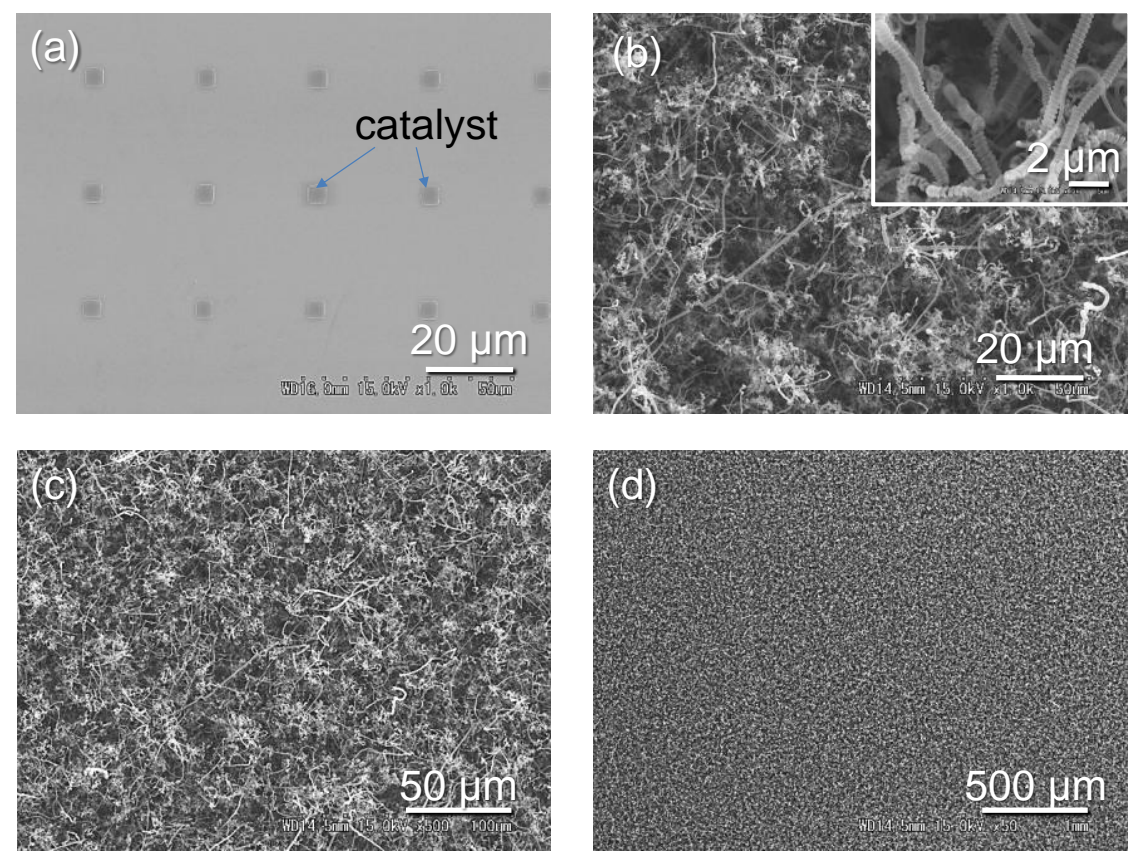

Figure 7 (a) Catalyst pattern on the substrate with a gap of $24 \mu \mathrm{m}$ for checker board pattern catalyst. (b)-(d) SEM images with different magnification of grown CNCs.

The optimization of the ratio of the gap and catalyst size is important to realize the high yield syntheses of CNCs. In our experiments, the optimum condition for the yield of CNCs is $2 \mu \mathrm{m}$ square with a gap of $2 \mu \mathrm{m}$ without significant reduction of CNC layer thickness. Note that we have intended to investigate the effect of the collision of catalyst, so that the optimization for high yield synthesis is the subject for further study. Consequently, suppression of the collision of growing CNCs at the early growth stage is extremely efficient for the smooth growth of CNCs. The highest purity (the ratio of cross sectional areas of CNC and carbon byproducts) of $88.7 \%$ is achieved with nearly isolated island pattern as shown in Figs. 6 and 7. We believe that this simple strategy to suppress the collision of the tip catalyst at $\mathrm{CNC}$ presents opportunities for the mass synthesis of CNCs. 


\section{Conclusions}

We investigated the effects of the collisions of the catalyst on the growing CNC tip on $\mathrm{CNC}$ growth using a thin film catalyst with a stripe pattern. The grown length at the gap separating the adjacent catalyst stripes along the substrate depends strongly on the gap distance, whereas the growth length on top is independent of the gap length, which indicates that the collision of each CNC inhibits the smooth growth of CNCs and forms the dense entangled and shortened CNC layer under the CNC grown layer because of the deactivation of the catalyst at the growing CNC tip. Results show that the well-controlled thin film catalyst with a checkerboard pattern $(2 \mu \mathrm{m}$ square) brings four-times-higher efficiency of catalyst without significant degradation of CNC yield and the suppression of carbon byproduct layer to $\sim 1 / 3$. We believe that this simple strategy to suppress the collision of the tip catalyst at growing CNCs might facilitate the mass production of CNCs.

\section{Acknowledgement}

This work was partially supported by the Osaka Prefecture Collaboration of Regional Entities for the Advancement of Technological Excellence, JST and by the Iketani Science and Technology Foundation. 


\section{References}

1. Davis WR, Slawson RJ, Rigby GR. An Unusual Form of Carbon. Nature. 1953;171(4356):756-.

2. Amelinckx S, Zhang XB, Bernaerts D, Zhang XF, Ivanov V, Nagy JB. A Formation Mechanism for Catalytically Grown Helix-Shaped Graphite Nanotubes. Science. 1994;265(5172):635-9.

3. Chen XQ, Zhang SL, Dikin DA, Ding WQ, Ruoff RS, Pan LJ, et al. Mechanics of a carbon nanocoil. Nano Lett. 2003;3(9):1299-304.

4. Hayashida T, Pan L, Nakayama Y. Mechanical and electrical properties of carbon tubule nanocoils. Physica B. 2002;323(1-4):352-3.

5. Pan LJ, Hayashida T, Zhang M, Nakayama Y. Field emission properties of carbon tubule nanocoils. Jpn J Appl Phys. 2001;40(3B):L235-L7.

6. Jiao J, Einarsson E, Tuggle DW, Love L, Prado J, Coia GM. High-yield synthesis of carbon coils on tungsten substrates and their behavior in the presence of an electric field. $\mathrm{J}$ Mater Res. 2003;18(11):2580-7.

7. Pan L, Konishi O, Tanaka H, Suekane O, Nosaka T, Nakayama Y. Effect of morphology on field emission properties of carbon nanocoils and carbon nanotubes. Jpn J Appl Phys. 2005;44(4A):1652-4.

8. Kanada R, Pan L, Akita S, Okazaki N, Hirahara K, Nakayama Y. Synthesis of multiwalled carbon nanocoils using codeposited thin film of Fe-Sn as catalyst. Jpn J Appl Phys. 2008;47(4):1949-51.

9. Motojima S, Chen X, Yang S, Hasegawa M. Properties and potential applications of carbon microcoils/nanocoils. Diam Relat Mater. 2004;13(11-12):1989-92.

10. Motojima S, Noda Y, Hoshiya S, Hishikawa Y. Electromagnetic wave absorption property of carbon microcoils in 12-110 GHz region. J Appl Phys. 2003;94(4):2325.

11. Liu L, He PG, Zhou KC, Chen TF. Microwave absorption properties of carbon fibers with carbon coils of different morphologies (double microcoils and single nanocoils) grown on them. J Mater Sci. 2014;49(12):4379-86.

12. Eguchi U, Takikawa H, Suda Y. Electromagnetic wave absorption characteristics of multiwalled carbon nanocoils. Jpn J Appl Phys. 2014;53(4).

13. Zhang M, Nakayama Y, Pan LJ. Synthesis of carbon tubule nanocoils in high yield using iron-coated indium tin oxide as catalyst. Jpn J Appl Phys. 2000;39(12A):L1242-L4.

14. Pan LJ, Zhang M, Nakayama Y. Growth mechanism of carbon nanocoils. J Appl Phys. 2002;91(12):10058-61.

15. Xu GC, Chen BB, Shiki H, Katsumata T, Takikawa H, Sakakibara T, et al. Parametric study on growth of carbon nanocoil by catalytic chemical vapor deposition. Jpn J Appl Phys. 2005;44(4A):1569-76.

16. Okazaki N, Hosokawa S, Goto T, Nakayama Y. Synthesis of carbon tubule nanocoils using Fe-In-Sn-O fine particles as catalysts. J Phys Chem B. 2005;109(37):17366-71.

17. Liu WC, Lin HK, Chen YL, Lee CY, Chiu HT. Growth of Carbon Nanocoils from K and Ag Cooperative Bicatalyst Assisted Thermal Decomposition of Acetylene. Acs Nano. 2010;4(7):4149-57.

18. Hirahara K, Nakayama Y. The effect of a tin oxide buffer layer for the high yield synthesis of carbon nanocoils. Carbon. 2013;56:264-70.

19. Li DW, Pan LJ. Necessity of base fixation for helical growth of carbon nanocoils. J Mater Res. 2012;27(2):431-9. 
20. Sun JY, Koos AA, Dillon F, Jurkschat K, Castell MR, Grobert N. Synthesis of carbon nanocoil forests on BaSrTiO3 substrates with the aid of a Sn catalyst. Carbon. 2013;60:5-15.

21. Gohara T, Takei K, Arie T, Akita S. In-situ optical microscopy observations of the growth of individual carbon nanocoils. J Vac Sci Technol B. 2014;32(3):031807-1-5. 


\section{Figure captions}

Figure 1(a) Schematic illustration of the arrangement of thin film catalyst to investigate the collision of the growing $\mathrm{CNCs}$, where a tri-layer catalyst was deposited on the $\mathrm{SiO}_{2} / \mathrm{Si}$ substrate. (b) Schematic illustration of cross-sectional view for grown CNCs around the catalyst.

Figure 2 Effect of gap distance dependence on CNC growth: (a) Gap distance dependence of CNC length grown between adjacent strips with several growth time. A straight line represents half of the gap distance. (b) Gap distance dependence of $\mathrm{CNC}$ length on top of the catalyst center with several growth time. (c) Temporal variation of the in-situ optical microscope image of growing CNCs around the different stripe gaps with $20 \mu \mathrm{m}$ and $40 \mu \mathrm{m}$.

Figure 3 Cross-sectional SEM image of stripe catalyst: (a) SEM image of the cross section around the stripe catalyst (scale bar is $50 \mu \mathrm{m}$ ). (b) Expanded view at the carbon byproduct layer consisting from dense and shortened CNCs (scale bar is $2 \mu \mathrm{m}$ ). (c) Expanded view of the carbon byproduct layer consisting of dense amorphous carbon. (d) Gap length dependence of the ratio of areas between $\mathrm{CNC}$ and byproduct measured from the cross sectional SEM image.

Figure 4 Growth model of CNCs: (a) immediately after the introduction of source gas, (b) nanoparticles and a-C layer formation after $10 \mathrm{~s}$, (c) very early stage of $\mathrm{CNC}$ formation on the catalyst surface, (d) beginning of collision of adjacent CNCs, (e) beginning of formation of shortened and dense fibrous $\mathrm{C}$ layer and of collision of CNCs at the gap, and (f) the gap is occupied by collided CNCs. 
Figure 5 Suppression of dense a-C and shortened and dense fibrous layer: cross-sectional and top view SEM images of CNCs grown from (a) and (d) a substrate fully covered with catalyst layer, (b) and (e) stripe catalyst with a width of $20 \mu \mathrm{m}$ and (c) and (f) checkerboard pattern with $2 \mu \mathrm{m}$ square. Yellow parts are insets of (a)-(c), showing the catalyst pattern on the substrate. All scale bars are $100 \mu \mathrm{m}$.

Figure 6 SEM images of grown CNCs with different gaps with (a) $2 \mu \mathrm{m}$, (b) $6 \mu \mathrm{m}$, and (c) 24 $\mu \mathrm{m}$ for checker board pattern catalyst. Arrows of bottom images indicate the substrate edges.

Figure 7 (a) Catalyst pattern on the substrate with a gap of $24 \mu \mathrm{m}$ for checker board pattern catalyst. (b)-(d) SEM images with different magnification of grown CNCs. 